ARTICLE

\title{
A cocktail of three virulent bacteriophages prevents Vibrio cholerae infection in animal models
}

\author{
Minmin Yen ${ }^{1,2, \star}$, Lynne S. Cairns ${ }^{1, \star} \&$ Andrew Camilli ${ }^{1}$
}

Effective prevention strategies will be essential in reducing disease burden due to bacterial infections. Here we harness the specificity and rapid-acting properties of bacteriophages as a potential prophylaxis therapy for cholera, a severely dehydrating disease caused by Vibrio cholerae. To this end, we test a cocktail of three virulent phages in two animal models of cholera pathogenesis (infant mouse and rabbit models). Oral administration of the phages up to $24 \mathrm{~h}$ before $V$. cholerae challenge reduces colonization of the intestinal tract and prevents cholera-like diarrhea. None of the surviving $V$. cholerae colonies are resistant to all three phages. Genome sequencing and variant analysis of the surviving colonies indicate that resistance to the phages is largely conferred by mutations in genes required for the production of the phage receptors. For acute infections, such as cholera, phage prophylaxis could provide a strategy to limit the impact of bacterial disease on human health.

\footnotetext{
${ }^{1}$ Department of Molecular Biology and Microbiology, Howard Hughes Medical Institute, Tufts University School of Medicine, 136 Harrison Avenue, Boston, Massachussetts 02111, USA. ${ }^{2}$ Program in Molecular Microbiology, Sackler School of Graduate Biomedical Sciences, Tufts University, Boston,

Massachussetts 02111, USA. * These authors equally contributed to this work. Correspondence and requests for materials should be addressed to A.C. (email: andrew.camilli@tufts.edu).
} 
C holera is an acute, severely dehydrating diarrheal disease caused by the water-borne bacterium Vibrio cholerae. Cholera remains a substantial global health burden and is endemic to many parts of Africa and Asia ${ }^{1}$. Recent widespread epidemics in disaster-stricken or war-torn countries such as Haiti $^{2}$ and Iraq $^{3}$ highlight the vulnerabilities of populations to sudden outbreaks. Current recommended preventatives include mass vaccinations with the World Health Organizationprequalified oral cholera vaccine ${ }^{4}$ and increased awareness of sanitation and hygiene practices ${ }^{5}$. However, access to clean water is difficult and vaccination campaigns require forethought and time for efficacy; both methods may not be logistically feasible for immediate protection in the event of an outbreak. Household transmission is a major contributor to the rapid spread of $V$. cholerae within communities. Household contacts of index cases often present with cholera symptoms 2-3 days after the initial patient becomes sick ${ }^{6}$. Therefore, there is currently an unmet need for a clinical intervention to stem the household spread of cholera by use of a rapid prophylactic treatment. Although chemoprophylaxis with antibiotics may effectively reduce cholera burden ${ }^{7}$, the World Health Organization does not recommend this practice due to the development and spread of drug-resistant bacteria (http://www.who.int/cholera/technical/ prevention/control/en/). Moreover, the broad-spectrum action of antibiotics would cause dysbiosis of the resident intestinal microbiota, which could put patients at risk of other intestinal infections.

There has recently been a renewed interest in the use of bacteriophages (phages) for environmental and clinical applications $^{8}$. In contrast to antibiotics, phages are specific in their targets and, because they are replicating viruses, are capable of auto-dosing, a phenomenon where phage replication increases their number and contributes to the dose. Previous attempts to use phages to prevent or treat cholera have produced mixed results. Dutta et al. ${ }^{9}$ showed that a single phage type given $1 \mathrm{~h}$ before $V$. cholerae challenge in an infant rabbit model prevented onset of cholera symptoms. Studies by Jaiswal et al. ${ }^{10}$ showed that a cocktail of five lytic bacteriophage types given 6 or $12 \mathrm{~h}$ before $V$. cholerae challenge in an adult rabbit model reduced diarrheal severity slightly but did not significantly lower the bacterial load; however, the same phage cocktail could successfully reduce the $V$. cholerae load when administered 6 or $12 \mathrm{~h}$ after challenge. A second study in adult mice also showed promise for treatment of cholera with a phage cocktail ${ }^{11}$. These studies suggest that a prophylaxis approach merits further, in-depth investigation.
We previously isolated three $V$. cholerae-specific, lytic (virulent) phages ICP1, ICP2 and ICP3 from rice-water stool samples of cholera patients in Bangladesh ${ }^{12}$. The receptors for ICP1 and ICP2 were identified as the lipopolysaccharide (LPS) $\mathrm{O} 1$ antigen $^{13}$ and the major outer membrane porin OmpU ${ }^{14}$, respectively, which are considered virulence factors of $V$. cholerae. The receptor for ICP3 is as yet unknown, although we hypothesize at least a partial role for the LPS O-antigen. We have recently shown that these virulent phages impose significant bactericidal pressure on $V$. cholerae during its natural course of infection in humans ${ }^{14}$. A cocktail comprising phages that target different receptors would reduce the likelihood of multi-phageresistant $V$. cholerae isolates in the surviving population. Therefore, we hypothesized that a cocktail of the three ICP phages may be used as a prophylaxis treatment to specifically target $V$. cholerae that transits into the small intestine to prevent signs of cholera in animal models of disease.

In this study, we show that orally applied, prophylactic use of the ICP cocktail reduces colonization by $V$. cholerae in the infant mouse model. The ICP cocktail also prevents the onset of cholera symptoms in the infant rabbit model when administered up to $24 \mathrm{~h}$ before $V$. cholerae challenge. This proof-of-principle study demonstrates the successful use of phage prophylaxis to prevent disease caused by a mucosal pathogen.

\section{Results}

The three-phage ICP cocktail kills $V$. cholerae in vitro. Initial in vitro killing time-course experiments provided support for the hypothesis that the three-phage ICP cocktail was more effective in killing $V$. cholerae than each phage in isolation (Fig. 1). Cultures of $V$. cholerae E7946 (AC53) were grown in (i) the absence of phage or in the presence of (ii) ICP1, (iii) ICP2, (iv) ICP3 or (v) the ICP cocktail, at a multiplicity of infection of 1 . Bacteria (Fig. 1a) and phage (Fig. 1b) titres were enumerated every hour for $8 \mathrm{~h}$ and then again at 12 and $24 \mathrm{~h}$. Although the bacterial population declined in all conditions initially, growth resumed for cultures grown in the presence of ICP1 or ICP3 within 4-6h, respectively (Fig. 1a). Cells incubated in the presence of ICP2 resumed growth more slowly, but reached the same density as the control within $24 \mathrm{~h}$. In contrast, cells grown with the ICP cocktail did not reach the same density as the no-phage control by the end of the experiment. Phage titres dropped but remained stable for all conditions over the course of the experiment (Fig. 1b). It is not surprising that $V$. cholerae was able to escape phage predation
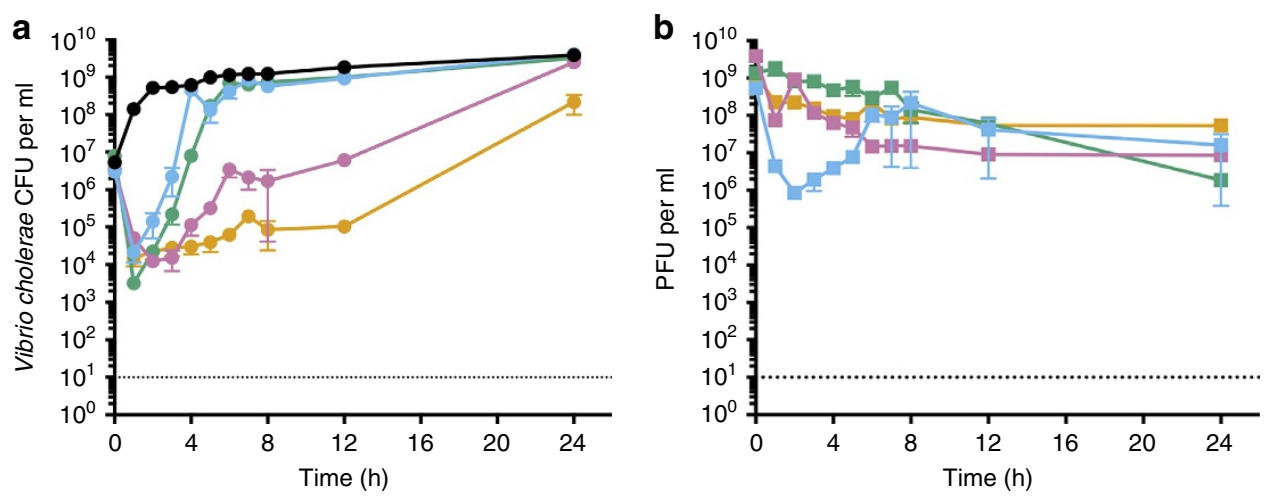

Figure 1 | The ICP cocktail kills V. cholerae in vitro. Growth curves of V. cholerae E7946 (AC53) in the absence and presence of phage predation. Cultures were grown in the absence of phage (black) or with ICP1 (blue), ICP2 (pink), ICP3 (green) or the ICP phage cocktail (orange) at an multiplicity of infection of 1. Samples of each culture were taken periodically and (a) enumerated for CFU per $\mathrm{ml}$ or (b) the phage fraction isolated and enumerated for PFU per ml by plaque assay. Data are plotted as the average of five independent replicates. Error bars represent the s.e.m. The dotted line represents the limit of detection. 
over time, given that the starting inoculum was high $\left(5 \times 10^{7}\right.$ colony-forming units (CFU)) and probably contained phageresistant mutants that were positively selected.

The ICP cocktail reduces mouse small intestinal colonization. The ICP cocktail was more effective than each phage alone in killing $V$. cholerae in vitro. To test whether this was also true in vivo, prophylaxis experiments were performed in the infant mouse model of $V$. cholerae colonization. We hypothesized that the ICP cocktail would be effective in preventing $V$. cholerae infection of the infant mouse small intestine. Mice were divided into five groups and received either (i) no phage, (ii) ICP1, (iii) ICP2, (iv) ICP3 or (v) the ICP cocktail. Mice were given phages (between $1 \times 10^{6}$ and $1 \times 10^{7}$ plaque-forming units (PFU)) by orogastric inoculation $3 \mathrm{~h}$ before infection with $5 \times 10^{5} \mathrm{CFU} V$. cholerae. After $24 \mathrm{~h}$, mice were killed, and $V$. cholerae and phages in the small intestine enumerated. As shown in Fig. 2a, the number of surviving $V$. cholerae cells in the small intestine was reduced by at least two orders of magnitude in all conditions where phage was administered, in comparison with the nontreated control, with ICP3 being the most successful single phage. No $V$. cholerae were detected in the intestinal homogenates of four out of five mice treated with the ICP cocktail $(P<0.01$, Kruskal-Wallis test with the Dunn's post-hoc multiple comparisons test). These data show that in comparison with ICP1 or ICP2 alone, ICP3 alone or the ICP cocktail is superior at preventing $V$. cholerae colonization. Phages were still detected in the small intestine at the end of the experiment (Fig. 2b), suggesting that these phages can survive and persist in the intestinal tract. Although the bacterial count medians of the ICP3 alone and the ICP cocktail groups are similar, we chose to proceed with the ICP cocktail to reduce the probability that phage-resistant $V$. cholerae isolates would survive.

Next, we aimed to test whether the ICP cocktail could still be effective when administered up to $24 \mathrm{~h}$ before $V$. cholerae infection. First, it was necessary to establish if phages could survive in the small intestine for long periods in the absence of their host bacterium. To do this, mice were dosed with phages (between $3 \times 10^{7}$ and $3 \times 10^{8} \mathrm{PFU}$ ) by oral gavage and killed after $3,6,12$ or $24 \mathrm{~h}$. Phages were enumerated from intestinal homogenates using plaque assays (Fig. $3 \mathrm{a}$ ). After $3 \mathrm{~h}$, the phage titres for ICP1 and ICP3 were still stable, whereas ICP2 titres had dropped $\sim 10$-fold. For all three phages, titres dropped only 10- to 100 -fold after $6-12 \mathrm{~h}$. After $24 \mathrm{~h}$, ICP1 and ICP2 were still retained at $\sim 10^{5} \mathrm{PFU}$ per small intestine, whereas the titre of ICP3 fell to between $10^{2}$ and $10^{4}$ PFU per small intestine. These data further show that the ICP phages can survive in the intestinal tract for at least $24 \mathrm{~h}$ in the absence of their host $V$. cholerae.

To test whether the ICP cocktail remained protective when given several hours before exposure to $V$. cholerae, infant mice were inoculated with the ICP cocktail 6,12 or $24 \mathrm{~h}$ before challenge with between $5 \times 10^{5}$ and $9 \times 10^{5} \mathrm{CFU} V$. cholerae. A control group did not receive phage. The data presented in Fig. $3 \mathrm{~b}$ show that the $6 \mathrm{~h}$ prophylaxis was most successful; the number of surviving $V$. cholerae cells fell at least three orders of magnitude, in comparison with the no-phage group, with no detectable $V$. cholerae for four of the seven mice that received phage $(P<0.0001$, Kruskal-Wallis test with the Dunn's post-hoc multiple comparisons test). The number of $V$. cholerae cells was also significantly lower for the $12 \mathrm{~h}$ group $(P<0.001)$, with four of these animals having no detectable $V$. cholerae at the end of the experiment. The number of $V$. cholerae cells was also reduced two orders of magnitude in the $24 \mathrm{~h}$ group. Furthermore, virulent phages were still detectable $24 \mathrm{~h}$ post-infection (Fig. $3 \mathrm{c}$ ).

To determine whether the ICP cocktail could limit colonization when mice are given a higher challenge dose, infant mice were inoculated with $1 \times 10^{8} \mathrm{CFU}$ of $V$. cholerae, a dose $\sim 200$-fold higher than the experiment shown in Fig. 3b,c. The data presented in Fig. $3 \mathrm{~d}$ show that the ICP cocktail was effective in reducing $V$. cholerae colonization of the small intestine by at least two orders of magnitude when administered at either 6,12 or $24 \mathrm{~h}$ before challenge. Phages were also still detected at $24 \mathrm{~h}$ post infection at similar levels in all three phage-treated groups (Fig. 3e). In concert, these data support the hypothesis that a phage prophylaxis approach can be used to prevent $V$. cholerae colonization of the small intestine.

$V$. cholerae could be isolated from intestinal homogenates of several of the mice dosed with the ICP cocktail (Figs 2 and 3). Owing to the complexity of infection within the gut, it is possible that these bacteria survive because either they did not encounter the ICP phages or these cells carry genetic mutations that allow them to escape phage predation. To determine the phage resistance profile of surviving cells, $V$. cholerae isolates from the
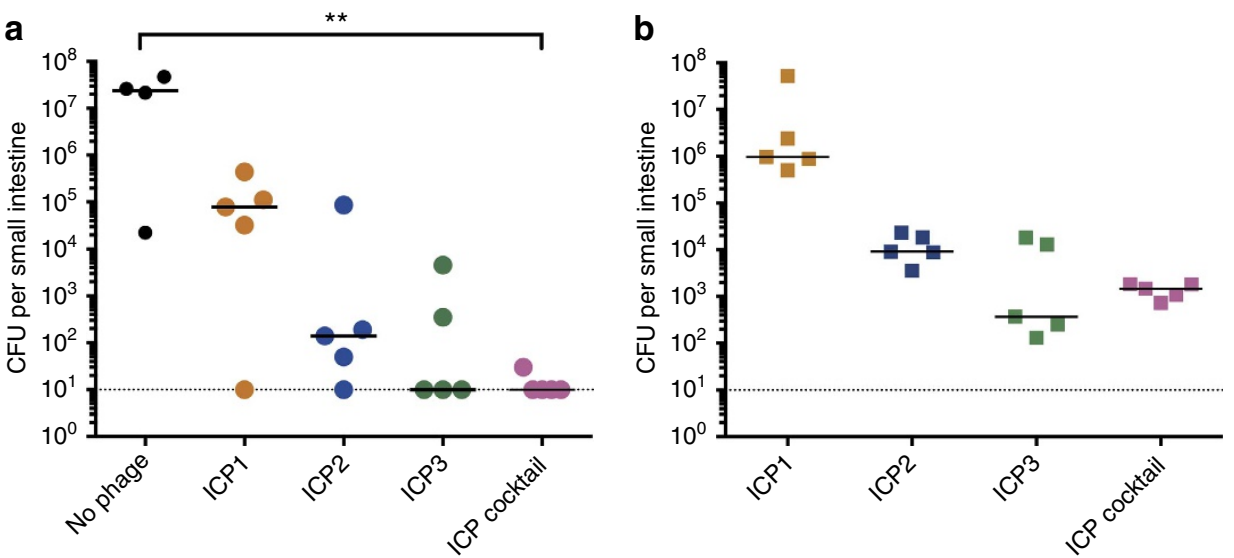

Figure 2 | Efficacy of the ICP cocktail and the ICP phages in reducing V. cholerae colonization of the infant mouse small intestine. Mice were dosed with between $1 \times 10^{6}$ and $1 \times 10^{7}$ PFU of the indicated phages $3 \mathrm{~h}$ before infection with $5 \times 10^{5} \mathrm{CFU}$ V. cholerae E7946 (AC53). A control group did not receive phage $(n=4)$. For each phage-treated group of animals, $n=5$. (a) V. cholerae that survived in the small intestine were enumerated as CFU per small intestine. (b) Phages that survived in the small intestine were enumerated as PFU per small intestine by plaque assay. The dotted line represents the limit of detection and the horizontal solid bar represents the median. Each square or circle represents one animal. Significance was calculated using the KruskalWallis test with the Dunn's post-hoc multiple comparisons test. ${ }^{\star \star} P=0.001-0.01$. 

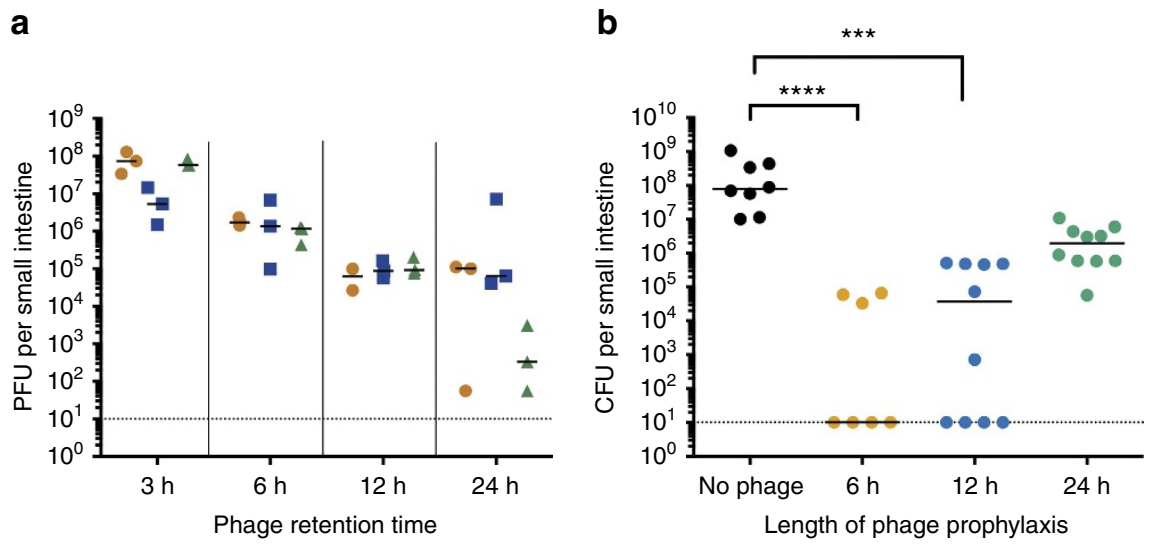

C

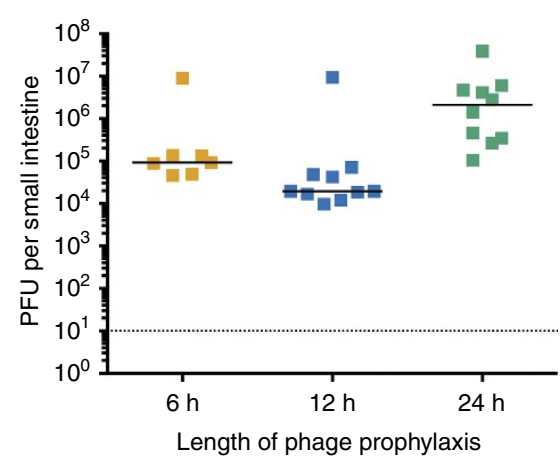

d

e

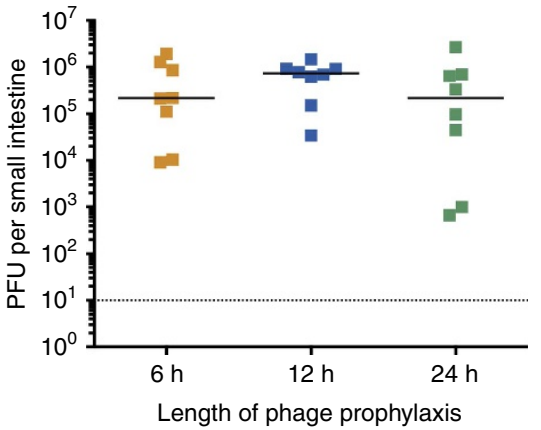

Figure 3 | V. cholerae burden in the infant mouse small intestine is reduced when the ICP cocktail is administered up to $24 \mathrm{~h}$ before infection. (a) Phages are retained in the infant mouse small intestine in the absence of the $V$. cholerae host. Mice were dosed with between $3 \times 10^{7}$ and $3 \times 10^{8}$ PFU of the indicated phage and killed after $3,6,12$ or $24 \mathrm{~h}$. Phages surviving in the small intestine were calculated by enumeration of PFU per small intestine from plaque assays. An orange circle represents ICP1, a blue square ICP2 and a green triangle ICP3. For each group, $n=3$. (b) Infant mice were dosed with between $2 \times 10^{5}$ and $4.8 \times 10^{5}$ PFU of the ICP cocktail either $6 \mathrm{~h}(n=7), 12 \mathrm{~h}(n=10)$ or $24 \mathrm{~h}(n=10)$ before infection with between $5 \times 10^{5}$ and $9 \times 10^{5}$ CFU V. cholerae E7946 (AC53). A control group did not receive phage $(n=8)$. Twenty-four hours after infection, the mice were killed and $V$. cholerae surviving in the small intestine enumerated by calculation of CFU per small intestine. (c) Phages surviving in the small intestine after the challenge described in $\mathbf{b}$ were enumerated by calculation of PFU per small intestine from plaque assays. (d) Infant mice were dosed with between $1 \times 10^{8}$ and $1.3 \times 10^{8} \mathrm{PFU}$ of the ICP cocktail $6 \mathrm{~h}(n=8), 12 \mathrm{~h}(n=8)$ or $24 \mathrm{~h}(n=8)$ before infection with a 'high-challenge dose' of $1 \times 10^{8}$ CFU V. cholerae E7946 (AC53). Twenty-four hours after infection, mice were killed and the $V$. cholerae surviving in the small intestine enumerated as CFU per small intestine, and phages enumerated as PFU per small intestine by plaque assay (e). The dotted line represents the limit of detection and the horizontal solid bar represents the median. Each square or circle represents one animal. Significance was calculated using the Kruskal-Wallis test with the Dunn's post-hoc multiple comparisons test. ${ }^{\star} P=0.01-0.05,{ }^{\star \star \star} P=0.0001-0.001$ and ${ }^{\star \star \star \star} P<0.0001$

experiment in Fig. 3b,c were randomly selected for colony purification and used in efficiency of plating (EOP) assays. The data are summarized in Supplementary Table 1 and detailed in Supplementary Data 1 . All isolates from mice that received the ICP cocktail 6 or $12 \mathrm{~h}$ before challenge were sensitive to all three ICP phages. The majority of isolates from mice given the ICP cocktail $24 \mathrm{~h}$ before challenge were sensitive to all three ICP phages; however, a small number showed differing ICP resistance phenotypes.

To uncover the genetic basis for resistance, $24 \mathrm{~V}$. cholerae isolates were analysed by whole-genome sequencing followed by variant analysis ${ }^{15-17}$. It was shown previously that slipped-strand mispairing in the poly-A tracts of O-antigen synthesis genes can result in abnormal $\mathrm{O}$-antigen and confer ICP1 resistance ${ }^{13}$. Consistent with this, the mutations in ICP1- and ICP3-resistant isolates were found in $\mathrm{O}$-antigen synthesis genes located on chromosome 1 of $V$. cholerae between open reading frames VC0240 (gmhD) and VC0269 (manA) ${ }^{18}$ (Supplementary Data 2). Although LPS mutations were a common source of resistance in these isolates, this is not of major concern given that strains carrying such mutations have previously been shown to be avirulent ${ }^{19-21}$. We have also shown previously that mutations in ompU and toxR confer ICP2 resistance ${ }^{14}$. In agreement, mutations in ICP2-resistant isolates were found in open reading frames VC0633 (ompU) or VC0984 (toxR) (Supplementary Data 2). Eight of the sequenced strains revealed no mutations to explain their phage-resistant phenotypes, most likely due to incomplete genome sequence coverage.

The ICP cocktail provides protection in the infant rabbit. To further assess the ability of the ICP cocktail to prevent cholera, experiments were undertaken in infant rabbits. In contrast to infant mice, infant rabbits infected by $V$. cholerae develop the profuse secretory diarrhea associated with cholera. Sickened animals suffer from dehydration and weight loss and show an accumulation of caecal fluid containing a high bacterial load 22 . First, to establish whether phages could be retained in the rabbit 
a

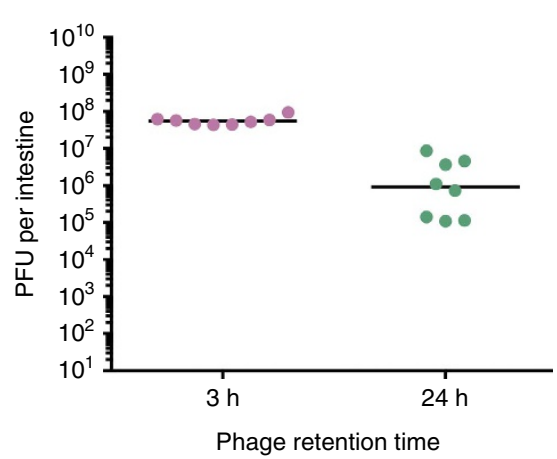

b

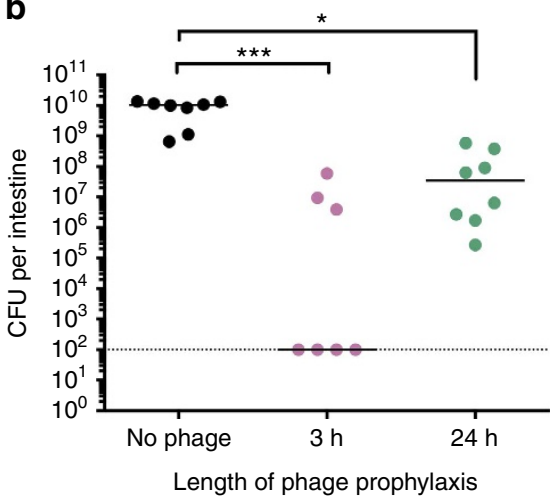

c

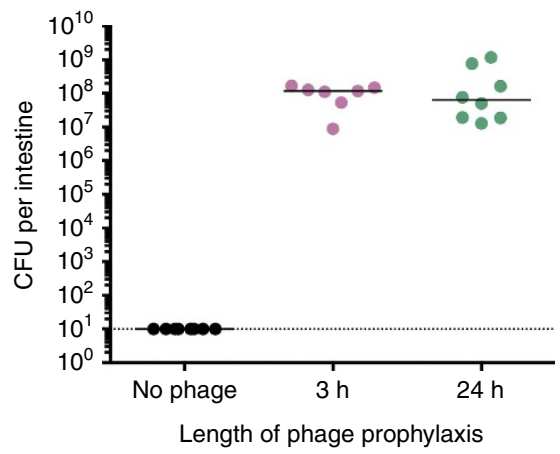

Figure 4 | Infant rabbits dosed with the ICP phage cocktail before $\boldsymbol{V}$. cholerae infection have a reduced bacterial load in the intestine. (a) Infant rabbits were dosed with $3 \times 10^{8} \mathrm{PFU}$ of the ICP phage cocktail and killed after $3 \mathrm{~h}(n=8)$ or $24 \mathrm{~h}(n=8)$. Phage retention in the intestine in the absence of the $V$. cholerae host was measured by enumeration of PFU per intestine. (b) Infant rabbits were dosed with between $4 \times 10^{9}$ and $8 \times 10^{9} \mathrm{PFU}$ of the ICP phage cocktail either $3 \mathrm{~h}(n=7)$ or $24 \mathrm{~h}(n=8)$ before infection with $5 \times 10^{8} \mathrm{CFU}$ V. cholerae E7946 (AC53). A control group $(n=8)$ did not receive phage. At the end of the infection period (either $12-14 \mathrm{~h}$ for the $\mathrm{V}$. cholerae only group, or $20 \mathrm{~h}$ after $\mathrm{V}$. cholerae infection for the phage-treated groups (see Methods for more details)), rabbits were killed and $V$. cholerae surviving in the intestine were enumerated as CFU per intestine. (c) Phages surviving in the intestine after the challenge described in $\mathbf{b}$ were enumerated as PFU per intestine. The dotted line represents the limit of detection and the horizontal solid bar represents the median. Each square or circle represents one animal. Significance was calculated using the Kruskal-Wallis test with the Dunn's post-hoc multiple comparisons test. ${ }^{\star} P=0.01-0.05$ and ${ }^{\star \star \star} P=0.0001-0.001$.

intestinal tract in the absence of $V$. cholerae, animals were dosed with the ICP cocktail alone $\left(3 \times 10^{8} \mathrm{PFU}\right)$. After 3 or $24 \mathrm{~h}$, the animals were killed and phages enumerated from intestinal homogenates. Even after $24 \mathrm{~h}, 10^{6}$ phages could be recovered from the intestine (Fig. 4a).

To determine whether the ICP cocktail could block infection by $V$. cholerae, phages (between $4 \times 10^{9}$ and $8 \times 10^{9} \mathrm{PFU}$ ) were orogastrically administered to two groups of infant rabbits either 3 or $24 \mathrm{~h}$ before challenge with $5 \times 10^{8} \mathrm{CFU} V$. cholerae. A control group did not receive phage. Animals were monitored for signs of cholera, specifically weight loss (Supplementary Fig. 1) and the presence of rice-water stool (also referred to here as cholera-like diarrhea). The no-phage control group showed cholera-like symptoms $12-14 \mathrm{~h}$ post infection, in line with previously published observations ${ }^{22}$, and were killed at this point. The caecum of each infected rabbit was distended and accumulated $\sim 0.5-1 \mathrm{ml}$ of fluid, which is further indicative of $V$. cholerae infection. Approximately $10^{9}-10^{10} \mathrm{CFU}$ were enumerated from caecal fluid (Supplementary Table 2) and intestinal homogenates (Fig. 4b) from this group.

$V$. cholerae were not detected for four of the seven rabbits that were dosed with ICP cocktail $3 \mathrm{~h}$ before infection (Fig. 4b), indicating that these animals cleared the infection. Between $10^{6}$ and $10^{8} \mathrm{CFU}$ of $V$. cholerae were obtained from intestinal homogenates from the remaining three rabbits in this group, giving a 10- to 100,000-fold decrease in comparison with the nontreated group (Fig. 4b, $P<0.001$, Kruskal-Wallis test with the Dunn's post-hoc multiple comparisons test). Between $\sim 10^{5}$ and $10^{9} \mathrm{CFU}$ of $V$. cholerae were enumerated from the intestines of animals dosed with phage $24 \mathrm{~h}$ before infection (Fig. 4b), presenting a 10- to 100,000 -fold decrease in bacterial load when compared with animals that received no phage $(P<0.05)$. It is important to note that despite the bacterial load observed, there was no evidence of rice-water stool or significant weight loss for rabbits in either of the phage-dosed groups up to $20 \mathrm{~h}$ after $V$. cholerae challenge (Supplementary Fig. 1). On dissection of these animals, $0.1 \mathrm{ml}$ of caecal fluid was obtained from one rabbit, which was dosed with phage $24 \mathrm{~h}$ before $V$. cholerae infection. The caeca of the other animals showed no signs of fluid accumulation (Supplementary Table 2). Between $10^{6}$ and $10^{9}$
PFU could be collected from the intestinal homogenates of phagetreated animals (Fig. 4c), indicating that phages persisted in the intestine over the course of the experiment. These observations suggest that the ICP cocktail protected against the signs of cholera over the duration of the experiment.

To determine whether $V$. cholerae that survived phage prophylaxis were sensitive or resistant to the ICP phages, $V$. cholerae isolates from each of the intestinal homogenates were randomly selected for colony purification and their phage resistance phenotype measured by EOP assays (summarized in Supplementary Table 3 and detailed in Supplementary Data 3). Thirty-nine per cent of isolates were resistant to both ICP1 and ICP3. No isolates were resistant to all three phages. To determine the genetic basis for resistance, whole-genome sequencing and variant analysis were conducted on 36 isolates, chosen due to their differing resistance phenotypes (Supplementary Data 4). Results were consistent with mutations found in ICP1- and ICP3-resistant isolates from the mouse experiment in Fig. 3. For 33 of these strains, there were mutations in O-antigen synthesis genes. For three strains, no mutations related to known phageresistance strategies were detected. We conclude from these data in two animal models that the leading source of phage resistance is mutation of the genes required for production of the phage receptors.

Collectively, these data suggest that the phage cocktail (i) is efficient at killing $V$. cholerae cells in vivo, (ii) reduces or prevents $V$. cholerae colonization of the intestine and (iii) provides protection against the onset of cholera-like diarrhoea in the infant rabbit model of cholera infection. Furthermore, isolates shed by the animal that were resistant to the ICP phages are expected to be avirulent based on past studies of O1-antigen mutants ${ }^{13}$, although virulence of these isolates has not yet been tested.

\section{Discussion}

Here we show that prophylactic administration of a $V$. choleraespecific phage cocktail is protective against cholera, by reducing both colonization and cholera-like diarrhoea in two animal models. We have shown that the ICP cocktail can successfully kill 
$V$. cholerae in vitro, prevent colonization of the infant mouse and preclude the onset of cholera-like diarrhoea in the infant rabbit. $V$. cholerae were isolated from intestinal homogenates of some phage-treated animals; however, the surviving population is smaller than observed from a wild-type $V$. cholerae infection (Figs 2-4). Moreover, we hypothesize that these surviving isolates may be unable to elicit acute infection, as many of the mutations that confer resistance to the ICP phages affect genes such as those involved in LPS synthesis (VC0240 to VC0269), which have been previously shown to be important for virulence. Crucially, at the end of these experiments, the population also remains sensitive to at least one of the three ICP phages. We suggest that the effectiveness of the ICP cocktail in killing $V$. cholerae cells is at least in part reliant on the concentration of phages that are in the intestine at the time of $V$. cholerae infection. The complex architecture of the intestinal tract probably presents a challenge to the phages' ability to access $V$. cholerae cells. The details of the interplay between the ICP phages, $V$. cholerae and the host remain to be elucidated.

Future optimization of the composition and dose of the cocktail could further improve its effectiveness. The ratio of each phage in the cocktail could be optimized and additional phages could be added to improve $V$. cholerae killing. Furthermore, as complete reduction of the bacterial load was only observed at shorter prophylaxis times, optimization of the timing for each dose is needed before human use. It is likely that an individual will be repeatedly exposed to $V$. cholerae; therefore multiple doses may be necessary to control the infection to a point where it can be completely eradicated.

Current phage therapy research is largely focused on treating ongoing infections. Our results highlight the potential of phage therapy in preventing infections. The data presented herein suggest that the premise of using a phage cocktail to prevent cholera warrants further investigation. We anticipate that a rapidacting phage prophylaxis approach would be best used in at-risk individuals, such as the household contacts of individuals who display cholera symptoms. By limiting spread within households, the overall burden of the disease could be reduced. The application of phages as prophylactic treatments for mucosal pathogens represents a fast and specific means by which to restrict the impact of bacterial infections on human health.

\begin{abstract}
Methods
Growth conditions and strains. Bacterial strains and phages used in this study are listed in Table 1. V. cholerae strains were grown in Luria-Bertani (LB) broth supplemented with $100 \mu \mathrm{g} \mathrm{ml}^{-1}$ streptomycin (Sm). The ICP cocktail comprises the Vibrio phages ICP1, ICP2 and ICP3 in equal number. These phages were previously isolated from Bangladeshi cholera patient rice-water stool samples ${ }^{12}$. ICP1 was isolated on strain AC4741, whereas ICP2 and ICP3 were isolated on AC53, an Sm-resistant isolate of E7946. All experiments were carried out using AC53 V. cholerae O1 El Tor strain E7946. Strains AC4653 and AC2846 were used in plaque assays as negative controls. ICP1 and ICP3 cannot plaque on AC4653, whereas ICP2 cannot plaque on AC2846.
\end{abstract}

Bacteriophage preparation. High-titre stocks of the ICP phages were prepared by growth on agar plates followed by polyethylene glycol (PEG) precipitation. Briefly, each phage was grown with the appropriate $V$. cholerae strain in soft agarose (LB broth supplemented with $0.3 \%$ agarose) overlays. Once confluent, overlays were incubated with STE buffer ( $100 \mathrm{mM} \mathrm{NaCl}, 10 \mathrm{mM}$ Tris and $1 \mathrm{mM}$ EDTA) overnight at $4{ }^{\circ} \mathrm{C}$ with gentle rocking to elute phage. The STE-phage solution was clarified by centrifugation, sterile-filtered and incubated with $1 \times$ PEG (4\% PEG $8000,0.5 \mathrm{M} \mathrm{NaCl}$ ) at $4^{\circ} \mathrm{C}$ for $1-3$ days, to allow for phage precipitation. Phages were collected by centrifugation at $10,000 \mathrm{~g}$ for $15 \mathrm{~min}$ at $4{ }^{\circ} \mathrm{C}$ and the phage pellet re-suspended in STE buffer. Phages were titred by plaque assay, as previously described $^{10}$.

In vitro phage-killing assay. Overnight cultures originating from single colonies of $V$. cholerae were diluted back to an $\mathrm{OD}_{600}$ of 0.05 in $50 \mathrm{ml} \mathrm{LB}$ supplemented with $100 \mu \mathrm{g} \mathrm{ml}{ }^{-1} \mathrm{Sm}$ and grown at $37^{\circ} \mathrm{C}$ with aeration. After $15 \mathrm{~min}$, phages were

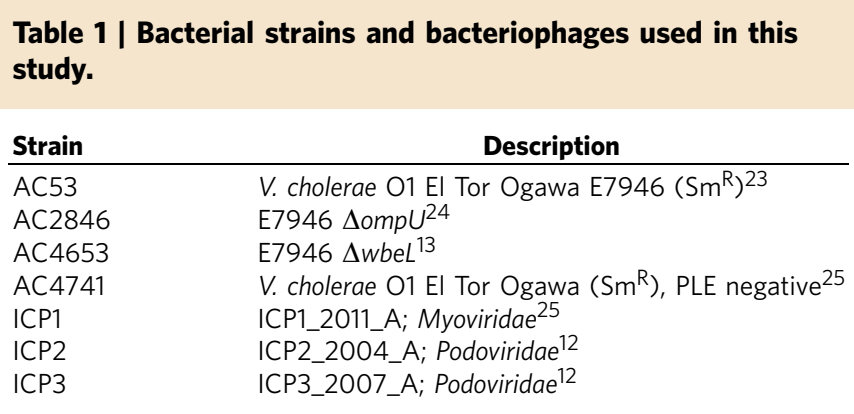

$\mathrm{PLE}, \mathrm{PICl}$-like element; $\mathrm{Sm}^{\mathrm{R}}$, streptomycin resistance.

added to each culture. At the indicated time-points, samples were collected to measure (i) CFU per $\mathrm{ml}$ and (ii) PFU per $\mathrm{ml}$.

Infection of infant mice with $\boldsymbol{V}$. cholerae. All animal experiments were in accordance with the rules of the Department of Laboratory Animal Medicine at Tufts University and the Institutional Animal Care and Use Committee. Four- and 5-day-old CD-1 infant mice (Charles River Laboratories) were infected. Each group of mice was comprised of animals from at least two different litters. Both male and female animals were used in this study. There was no observable correlation between sex of the animal and outcome of each experiment. Numbers of mice used in each condition in each experiment are listed in the legends of Figs 2 and 3. These mice were not tested for the presence of resident $V$. cholerae phages as the animals were not previously exposed to $V$. cholerae. As such, we do not predict that they will harbour any $V$. cholerae-specific phages in their microbiome.

Infant mice in phage-treated groups were orogastrically dosed with phage diluted in $2.5 \%$ sodium bicarbonate. Infant mice in cholera-treated groups received ca. $10^{5}$ CFU ('normal' infectious dose) or ca. $10^{8} \mathrm{CFU}$ ('high' infectious dose) of $V$. cholerae diluted in $2.5 \%$ sodium bicarbonate. As phages were given at least $3 \mathrm{~h}$ before bacteria, any multiplicity of infection calculated would be inaccurate. Instead, the titres of bacteria and phage inoculated into animals were calculated from the input materials and the ranges specified in the figure legends. Mice were killed $24 \mathrm{~h}$ post infection, the longest infection time permitted in our protocol. Small intestines were dissected and homogenized in LB broth supplemented with $20 \%$ glycerol. Cells were serially diluted and plated on LB agar supplemented with $100 \mu \mathrm{g} \mathrm{ml}^{-1} \mathrm{Sm}$ and CFU per small intestine was calculated. To extract phage from small intestine homogenates, an aliquot of the intestinal homogenate was treated with chloroform and centrifuged at $10,000 \mathrm{~g}$ for $5 \mathrm{~min}$. The supernatant was collected and used in plaque assays, to allow for calculation of PFU per small intestine. To assess the resistance profile of $V$. cholerae cells that survive phage prophylaxis, up to ten colonies were randomly picked per animal for use in EOP assays with ICP1, ICP2 and ICP3.

Infection of infant rabbits with $\boldsymbol{V}$. cholerae. Three-day-old New Zealand White infant rabbits (Charles River Laboratories) were used in this study. Each group of rabbits comprises animals from at least two different litters. Both male and female animals were used in this study. There was no observable correlation between sex of the animal and outcome of each experiment. Infant rabbits in phage-treated groups were orogastrically dosed with phage diluted in $2.5 \%$ sodium bicarbonate. Three hours before infection with $V$. cholerae, infant rabbits were pre-treated with ranitidine hydrochloride to reduce stomach acidity (Caraco Pharmaceutical Laboratories) by intraperitoneal injection ( $2 \mu \mathrm{g}$ per gram body weight). Infant rabbits were infected with $5 \times 10^{8} \mathrm{CFU} V$. cholerae AC53 diluted in $2.5 \%$ sodium bicarbonate. As for the mice experiments, exact titres of bacteria and phage inoculated were calculated from the input material and a range is detailed in the figure legends. Animals were weighed at the start of the experiment and periodically throughout the course of the infection. Percentage body weight was calculated by dividing body weight at the end of the infection period by body weight at the start. Animals were killed $12-20 \mathrm{~h}$ post infection. Infant rabbits that suffer from cholera typically lose $10-15 \%$ of their body weight within $12-14 \mathrm{~h}$ and are killed at this time. Infant rabbits that lost $<10 \%$ of their body weight are killed $20 \mathrm{~h}$ post infection, in accordance with our Institutional Animal Care and Use Committee protocol. This time restriction is necessary, as infants cannot be placed back with their mother after V. cholerae infection due to culling behaviour and so are without food for the infection period.

After dissection, the intestines of each rabbit were homogenized in LB broth supplemented with $20 \%$ glycerol. Caecal fluid, if present, was collected with a $1 \mathrm{ml}$ syringe. Caecal fluid and intestinal homogenates were serially diluted and plated on $\mathrm{LB}$ agar containing $100 \mu \mathrm{g} \mathrm{ml}^{-1} \mathrm{Sm}$ for calculation of CFU per intestine. Phages were collected from homogenates as described for infection of infant mice. Surviving $V$. cholerae colonies were colony-purified and assessed by EOP assays for resistance to ICP phages. 
EOP assays. EOP assays were performed for isolates that survived phage predation. Each phage was titered on the isolate, on AC53 and also a phage-resistant strain as a negative control $(\Delta w b e L$ AC4653 for ICP1 and ICP3; $\Delta o m p U$ AC2846 for ICP2). The EOP was calculated by dividing the titre of the phage on the animal isolate by the titre of the phage on AC53. The limit of detection was $1 \times 10^{-6}$. Isolates were described as resistant when the EOP was $<1 \times 10^{-6}$, sensitive when the EOP was $>1 \times 10^{-1}$ or partially sensitive when the EOP was between these values.

Sequence analysis of phage-resistant isolates. $V$. cholerae genomic DNA was extracted using a DNeasy Blood \& Tissue Kit (Qiagen). Whole-genome libraries were prepared for single-end 150-bp sequencing using the Nextera XT DNA Library Preparation Kit (Illumina). Sequencing was conducted at the Tufts University Core Facility using an Illumina HiSeq 2500. Genomes were assembled using CLC Genomics Workbench 8 software and aligned to the $V$. cholerae O1 N16961 reference genome ${ }^{13}$. To determine the mutations that may confer phage resistance, variant analysis was performed on mapped reads with a frequency threshold of $51 \%$. Results were compared with AC53 variants ${ }^{14}$ to remove those found in the wild-type inoculum. A surviving isolate that was determined to be sensitive to all three ICP phages was sequenced and resulting variants were also removed from resistant isolates variant analyses.

Data availability. The authors declare that the data supporting the findings of this study are available within the paper and its Supplementary Information files.

\section{References}

1. Zuckerman, J. N., Rombo, L. \& Fisch, A. The true burden and risk of cholera: implications for prevention and control. Lancet Infect. Dis. 7, 521-530 (2007).

2. Luquero, F. J. et al. Mortality rates during cholera epidemic, Haiti, 2010-2011. Emerg. Infect. Dis. 22, 410-416 (2016).

3. Bagcchi, S. Cholera in Iraq strains the fragile state. Lancet Infect. Dis. 16, 24-25 (2016).

4. Qadri, F. et al. Efficacy of a single-dose, inactivated oral cholera vaccine in Bangladesh. N. Engl. J. Med. 374, 1723-1732 (2016).

5. Taylor, D. L., Kahawita, T. M., Cairncross, S. \& Ensink, J. H. The impact of water, sanitation and hygiene interventions to control cholera: a systematic review. PLoS ONE 10, e0135676 (2015).

6. Harris, J. B. et al. Susceptibility to Vibrio cholerae infection in a cohort of household contacts of patients with cholera in Bangladesh. PLoS Negl. Trop. Dis. 2, e221 (2008).

7. Reveiz, L. et al. Chemoprophylaxis in contacts of patients with cholera: systematic review and meta-analysis. PLoS ONE 6, e27060 (2011).

8. Wittebole, X., De Roock, S. \& Opal, S. M. A historical overview of bacteriophage therapy as an alternative to antibiotics for the treatment of bacterial pathogens. Virulence 5, 226-235 (2014).

9. Dutta, N. K. \& Panse, M. V. An experimental study on the usefulness of bacteriophage in the prophylaxis and treatment of cholera. Bull. World Health Organ. 28, 357-360 (1963).

10. Jaiswal, A., Koley, H., Ghosh, A., Palit, A. \& Sarkar, B. Efficacy of cocktail phage therapy in treating Vibrio cholerae infection in rabbit model. Microbes Infect. 15, 152-156 (2013).

11. Jaiswal, A., Koley, H., Mitra, S., Saha, D. R. \& Sarkar, B. Comparative analysis of different oral approaches to treat Vibrio cholerae infection in adult mice. Int. J. Med. Microbiol. 304, 422-430 (2014).

12. Seed, K. D. et al. Evidence of a dominant lineage of Vibrio cholerae-specific lytic bacteriophages shed by cholera patients over a 10-year period in Dhaka, Bangladesh. mBio 2, e00334-00410 (2011).

13. Seed, K. D. et al. Phase variable $\mathrm{O}$ antigen biosynthetic genes control expression of the major protective antigen and bacteriophage receptor in Vibrio cholerae O1. PLoS Pathog. 8, e1002917 (2012).

14. Seed, K. D. et al. Evolutionary consequences of intra-patient phage predation on microbial populations. eLife 3, e03497 (2014).

15. Heidelberg, J. F. et al. DNA sequence of both chromosomes of the cholera pathogen Vibrio cholerae. Nature 406, 477-484 (2000).
16. Lazinski, D. W. \& Camilli, A. Homopolymer tail-mediated ligation PCR: a streamlined and highly efficient method for DNA cloning and library construction. BioTechniques 54, 25-34 (2013).

17. Heidelberg, J. F. et al. DNA sequence of both chromosomes of the cholera pathogen Vibrio cholerae. Nature 406, 477-483 (2000).

18. Chatterjee, S. N. \& Chaudhuri, K. Lipopolysaccharides of Vibrio cholerae II. Genetics of biosynthesis. Biochim. Biophys. Acta 1690, 93-109 (2004).

19. Kamp, H. D., Patimalla-Dipali, B., Lazinski, D. W., Wallace-Gadsden, F. \& Camilli, A. Gene fitness landscapes of Vibrio cholerae at important stages of its life cycle. PLoS Pathog. 9, e1003800 (2013).

20. Pritchard, J. R. et al. ARTIST: high-resolution genome-wide assessment of fitness using transposon-insertion sequencing. PLoS Genet. 10, e1004782 (2014).

21. Fu, Y., Waldor, M. K. \& Mekalanos, J. J. Tn-Seq analysis of Vibrio cholerae intestinal colonization reveals a role for T6SS-mediated antibacterial activity in the host. Cell Host Microbe 14, 652-663 (2013).

22. Abel, S. \& Waldor, M. K. Infant rabbit model for diarrheal diseases. Curr. Protoc. Microbiol. 38 6A 6 1-15 (2015).

23. Levine, M. M. et al. The pathogenicity of nonenterotoxigenic Vibrio cholerae Serogroup 01 Biotype EI Tor isolated from sewage water in Brazil. J. Infect. Dis. 145, 296-299 (1982)

24. Schild, S., Nelson, E. J. \& Camilli, A. Immunization with Vibrio cholerae outer membrane vesicles induces protective immunity in mice. Infect. Immun. 76, 4554-4563 (2008).

25. Seed, K. D., Lazinski, D. W., Calderwood, S. B. \& Camilli, A. A bacteriophage encodes its own CRISPR/Cas adaptive response to evade host innate immunity. Nature 494, 489-491 (2013).

\section{Acknowledgements}

We thank the Tufts University Core Facility for sequencing support. This work was supported by U.S. National Institutes of Health grant AI055058 to A.C. A.C. is a Howard Hughes Medical Institute Investigator. We thank members of the Camilli lab for useful discussions.

\section{Author contributions}

M.Y., L.S.C. and A.C. designed and performed experiments, and wrote the manuscript.

\section{Additional information}

Supplementary Information accompanies this paper at http://www.nature.com/ naturecommunications

Competing financial interests: M.Y., L.S.C. and A.C. have filed a provisional application, application number 62-316704, with the US Patent and Trademark Office on this work

Reprints and permission information is available online at http://npg.nature.com/ reprintsandpermissions/

How to cite this article: Yen, M. et al. A cocktail of three virulent bacteriophages prevents Vibrio cholerae infection in animal models. Nat. Commun. 8, 14187 doi: 10.1038/ncomms14187 (2017).

Publisher's note: Springer Nature remains neutral with regard to jurisdictional claims in published maps and institutional affiliations.

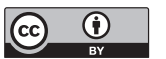

This work is licensed under a Creative Commons Attribution 4.0 International License. The images or other third party material in this article are included in the article's Creative Commons license, unless indicated otherwise in the credit line; if the material is not included under the Creative Commons license, users will need to obtain permission from the license holder to reproduce the material. To view a copy of this license, visit http://creativecommons.org/licenses/by/4.0/

(C) The Author(s) 2017 